\title{
A Multidimensional Impact Model for the Discovery of Extraterrestrial Life ${ }^{1}$
}

\author{
(v3.0) \\ Clément Vidal \\ Center Leo Apostel \\ Vrije Universiteit Brussel (Free University of Brussels) \\ Krijgskundestraat 33, 1160 Brussels, Belgium \\ Phone +32-2-640 6737 | Fax +32-2-6440744 \\ http://www.clemvidal.com \\ contact@clemvidal.com
}

Vidal, C. 2015. "A Multidimensional Impact Model for the Discovery of Extraterrestrial Life" In The Impact of Discovering Life Beyond Earth, edited by Steven J. Dick. Cambridge: Cambridge University Press.

\begin{abstract}
:
I first argue that the extraterrestrials we will find will not communicate, for the simple reason that they would likely be either immensely inferior or immensely superior to us. Then, I argue that the discovery of extraterrestrials will be slow, like other previous major scientific discoveries. I introduce a multidimensional model to assess and to prepare the impact of discovering extraterrestrial life. Twenty-six dimensions are introduced, illustrated with spider diagrams, which cover both what extraterrestrial might look like, and how humans may react.
\end{abstract}

Keywords: Astrobiology, extraterrestrial life, SETI, impact of discovery, slow discovery, Rio scale, worldview, spider diagram.

\section{Contents}

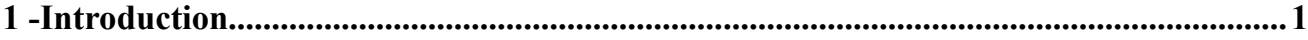

2 -Why extraterrestrials will not communicate....................................................................... 2

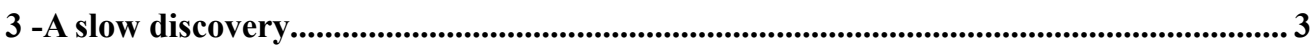

4 -A multidimensional impact model................................................................................... 3

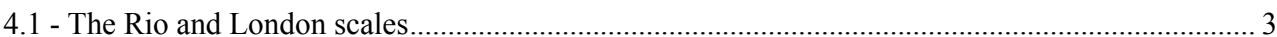

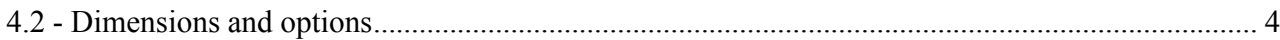

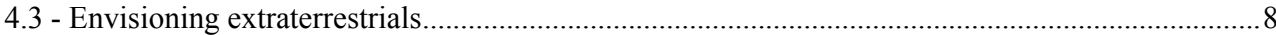

4.4 - Envisioning humanity's reaction: objective dimensions .................................................

4.5 - Envisioning humanity's reaction: intersubjective dimensions ...................................................... 11

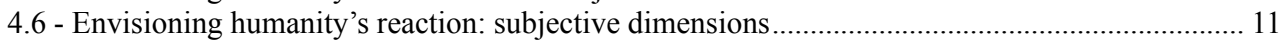

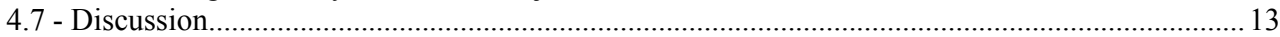

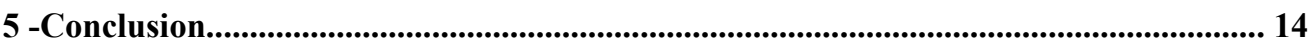

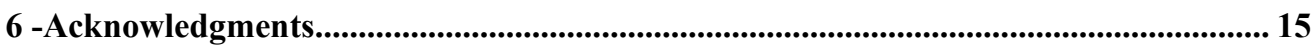

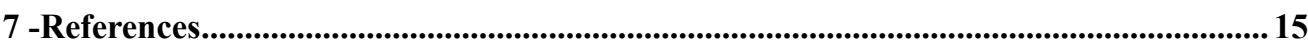

\section{1 - Introduction}

Most science fiction books and movies depict extraterrestrials (ETs) that are similar to us in many ways. They are at our scale, have eyes, limbs, body symmetries. But what if, the day we find ETs, they don't look like us? What if they are so different that no communication is possible? How would it impact our worldviews to find non-communicative ETs?

I first argue that we will most likely find microbial life or stellar civilizations, but nothing in-between. Then, I show that the discovery of ET life will most likely be very slow, taking years or decades. Finally, to prepare for discovery, I propose a multidimensional impact model.

1 Note that a previous version of this paper was entitled: Silent Impact: Why the Discovery of Extraterrestrial Life Should be Silent. 


\section{2 - Why extraterrestrials will not communicate}

The principle of mediocrity is fundamental in astrobiology. It says that "we should assume ourselves to be typical in any class that we belong to, unless there is some evidence to the contrary" (Vilenkin 2011). Applied to our position in space in the universe, it means that our solar system, our galaxy and possibly our universe -if there is a multiverse- are typical. They are not central or special in any way. This insight is well known and well assimilated, and is also known as the Copernican principle. However, what if we apply it to our position in time?

If we map our position in time according to the Kardashev (1964) scale (Fig. 1), we can see that we are in an extremely short transition phase from technical impotence to technical omnipotence. Indeed, the exponential growth of our energy consumption is very recent on evolutionary time scales, and will be limited when and if we are able to harness the energetic output of the Sun.

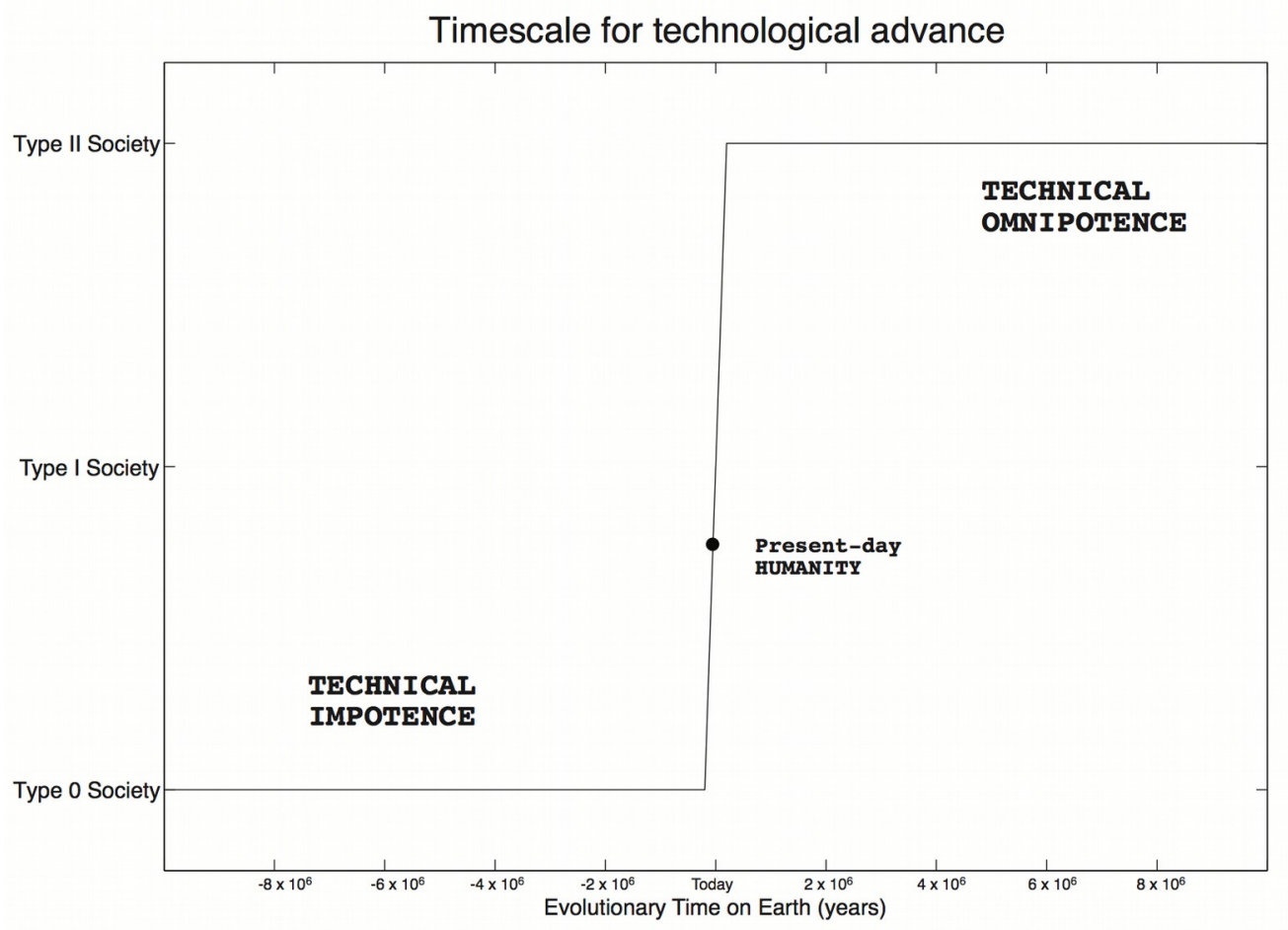

Figure 1 - From technical impotence to technical omnipotence. We are most likely to find microbial (Type 0) or stellar (Type II) extraterrestrials, but nothing in-between.

(Freitas Jr 1979, sec. 25.2.1)

If our position in time is typical, it means that we have most chances to find either microbial life (left-side of the step function), or stellar civilizations (right-side of the step function). It is extremely unlikely that we would find a civilization that is also in the short transition phase that we are in.

This difference in levels of development further leads to the argument that ETs will not communicate. If they are microbial, they will have not more to say than their biological organization can reveal (which is still very exciting for astrobiologists). If they are stellar civilizations, there might be ethical barriers to communication, as the prime directive in Star Trek which prohibits advanced civilizations to interfere with the development of lesser advanced ones. Of course, it is not an absolute rule, and there may be alien anthropologists who would want to study and communicate with alien races in the galaxy. 
However, the difference in development also brings a motivational barrier to communication. Why don't we spend time whispering to bacteria or plants that $E=m c^{2}$ ? It doesn't make sense to even try because it's simply a waste of time. In the same way, from the perspective of an advanced stellar civilization, it would probably make no sense to spend time and efforts to try to communicate to a relatively primitive and transitioning civilization such as our own.

There is a tradition of searching for non-communicative ETs, through searching for technological manifestations and artifacts (see e.g. Dyson 1960; 1966; Freitas Jr and Valdes 1985; Beech 2008; Bradbury, Ćirković, and Dvorsky 2011; Wright et al. 2014; Vidal 2014a, chap. 9). But how do we prove the existence of advanced ETs if we can not communicate with them?

\section{3 - A slow discovery}

The ideal SETI (search for extraterrestrial intelligence) scenario occurs if we find a signal easy to observe and to decode, clearly stating the existence of another ET civilization.

The issue of proving the presence of ETs is much more difficult if they do not communicate. In this case, we need to compare predictions and explanations hypothesizing that a phenomenon is purely physical, with predictions and explanations hypothesizing that the same phenomenon is living. The living or non-living model which predicts and explains most would gradually be favored. History shows that claims of non-communicative ET life were ambiguous for long periods of time. For example, the hypothesis of microfossils on the Martian meteorite ALH84001 (McKay et al. 1996) have fueled controversies which still are discussed today (see e.g. Wainwright 2014). Turning to more advanced ET life, take the now amusing idea that there are artificial canals on Mars. It was championed by Percival Lowell in the $19^{\text {th }}$ century, but was actually ambiguous for 20 to 70 years (Steven J. Dick 1996, $78)$ !

More generally, previous discoveries in astronomy constitute an extended process (Steven J. Dick 2013), and scientific revolutions took decades to be fully appreciated (e.g. Kuhn 1957; 1970). There is no reason the discovery of ET life should be different.

\section{4 - A multidimensional impact model}

\section{1 - The Rio and London scales}

Two scales have been proposed to assess the social impact of discovering ETs. The Rio scale attempts "to quantify the social impact" of a discovery of extraterrestrial intelligence (ETI), and the London scale has the same purpose for a discovery of extraterrestrial life (ETL) (Almár 2011). They have the merit of being simple, but too much so. Let us briefly examine limitations of these scales.

First, the distinction between the Rio and London scale assumes that the distinction between ETL and ETI will be clear cut, and unambiguous. This is of course not guaranteed, for even on Earth it is a contentious issue to decide where simple life stops and intelligence starts.

Second, these scales have much too few dimensions. Only three dimensions are introduced (class of phenomenon, discovery type and distance) which, when calculated, provide an output number between 0 and 10, representing the significance of the discovery. Giving a result in one single 
number is similar to other scales, such as Richter's, which assesses the strength of an earthquake. This unidimensional number could give a first approximation to how important the discovery is, and such a simplification might be useful for the media to communicate with the general public. However, a number between 0 and 10 will quickly prove too simplistic and people will want to know more. By contrast, I shall propose below a richer model, with 26 dimensions.

Third, as Shenkel (in Almár 2001) has noticed, using this approach immediately associates the discovery of ETs with a danger, like an earthquake or an asteroid impacting the Earth. But discovering ETs could as well be the greatest opportunity for humanity to grow and learn.

Fourth, Almár (2001) wrote:

I would like to emphasize that the announcements of putative discoveries we are discussing as well as the circumstances we are ranking are, or will be, most likely connected with physics and astronomy - not social sciences.

This is extremely surprising, if not self-contradictory, to aim to assess the social impact without considering social sciences! By contrast, Harrison's $(1997,244)$ book, one of the most comprehensive treatments of the subject matter, argues that "even the most clear and detailed announcement will elicit a variety of responses".

Fifth, the one-number outcome implicitly assumes the impact will be uniform for all humans, cultures, religions, and all domains of knowledge and action. This is consistent with the proposition of ignoring social sciences... but can we really and safely ignore the complexities of human beings and societies?

In preparing for discovery there are actually two equally challenging issues: to envison what ETs will be like, and to envision the reactions of humans. Indeed, an impact is the meeting point of two components, in this case, ETs and humans.

\section{2 - Dimensions and options}

The most objective way to study an impact is to be outside of it. This seemingly simple remark is of fundamental importance. In my research, I often try to take a "meta" perspective, which leads to new insights (see Vidal 2014a). It simply means to apply the concept to itself. What could it mean to take the perspective of "extraterrestrials of extraterrestrials"? It means to imagine not only what would happen from our perspective, or from the perspective of ETs involved; we should also imagine how a third ET civilization would analyze our impact with an ET life form or civilization.

The 26 dimensions are summarized in three spider diagrams (Fig. 2-4), and the options are laid out in Table 1. To choose the dimensions, I drew inspiration mostly from the Rio scale, and from Harrison's (1997) book. 
(1) Distance

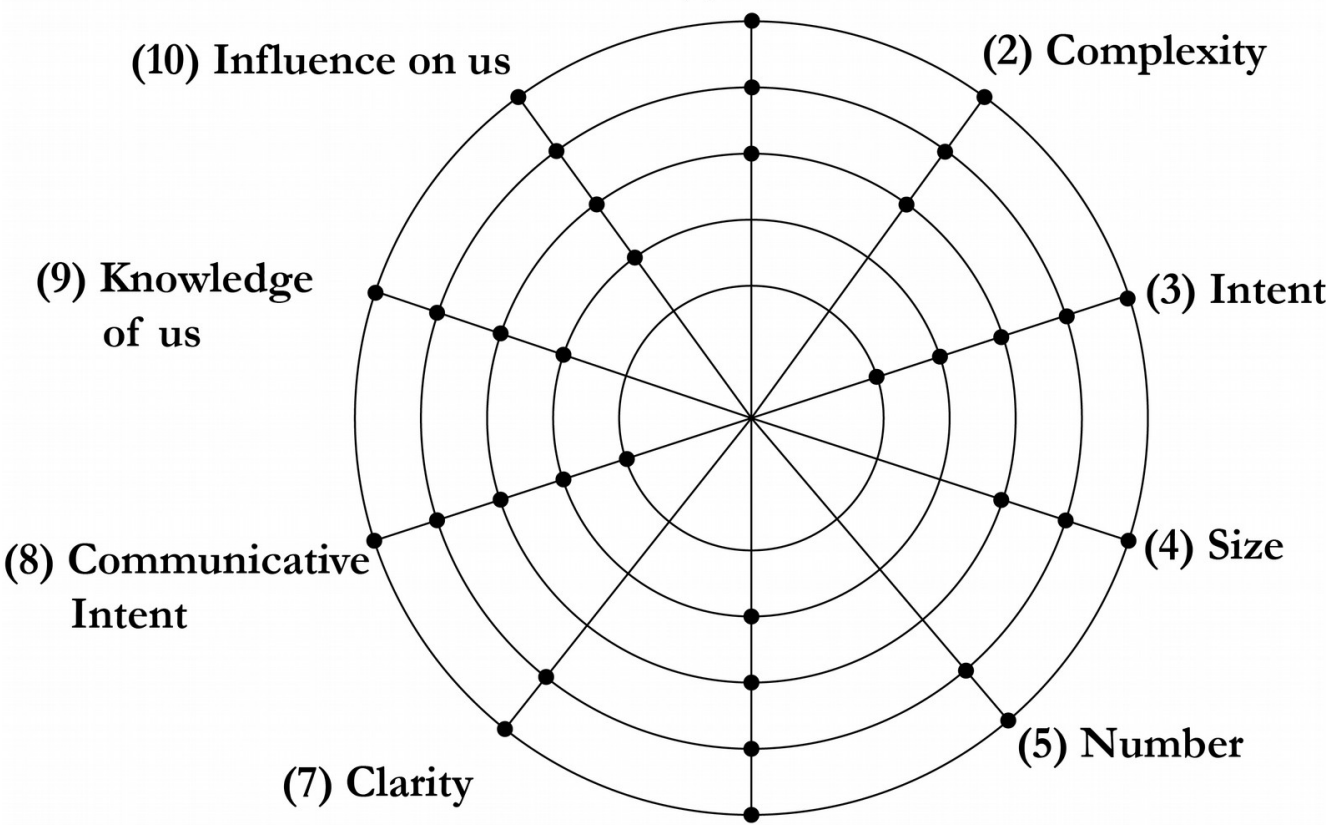

(6) Living state

Figure 2 - Ten dimensions of impact when envisioning extraterrestrials. Each radius represents one dimension, and each dot represents one option. The options are listed in Table 1.

\section{(a) Universalization of knowledge}

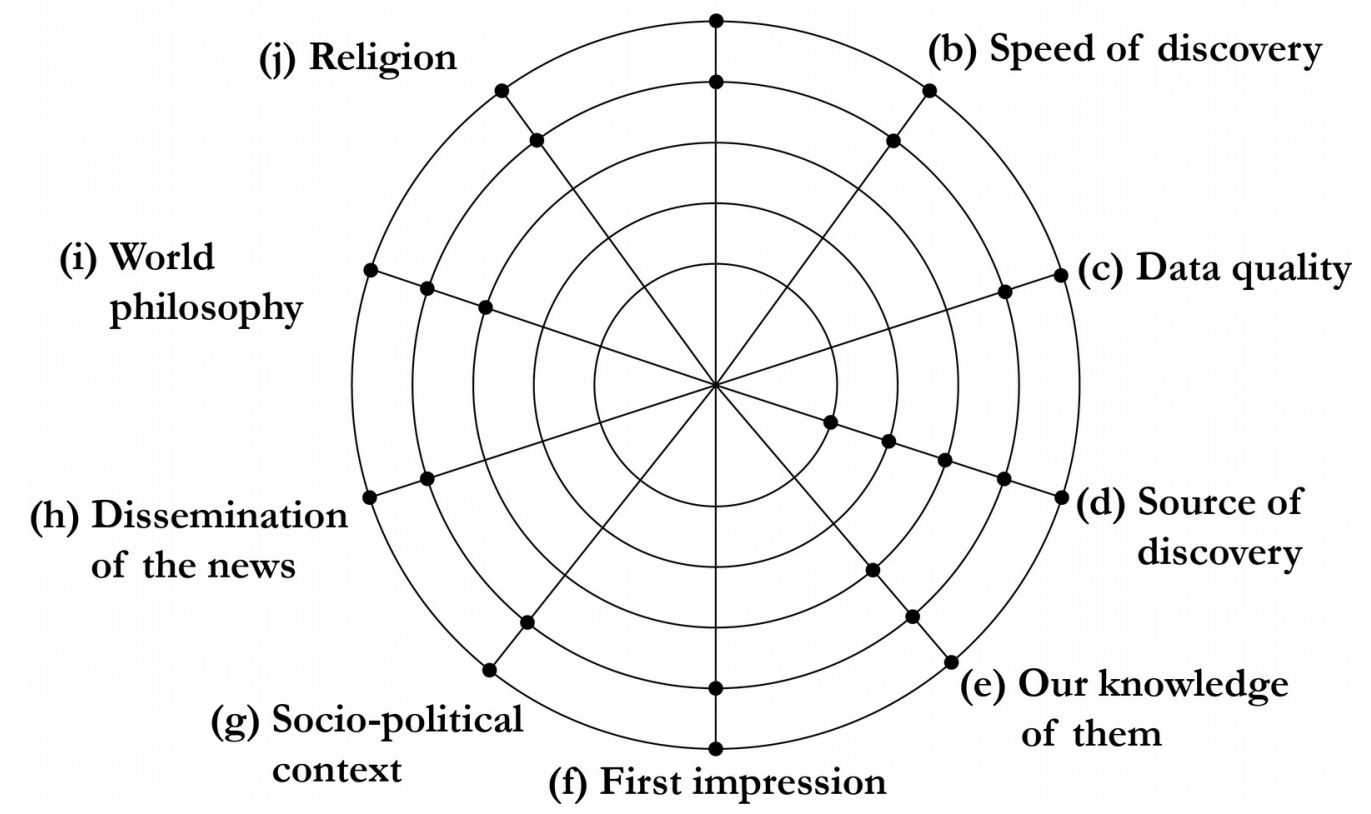

Figure 3 - Ten dimensions of impact when envisioning humanity. Five dimensions of impact (a-e) concern objective aspects of a putative discovery; and five dimensions (f-j) deal with intersubjective or social aspects. Subjective dimension are in Fig. 4, and all the options are listed in Table 1. 


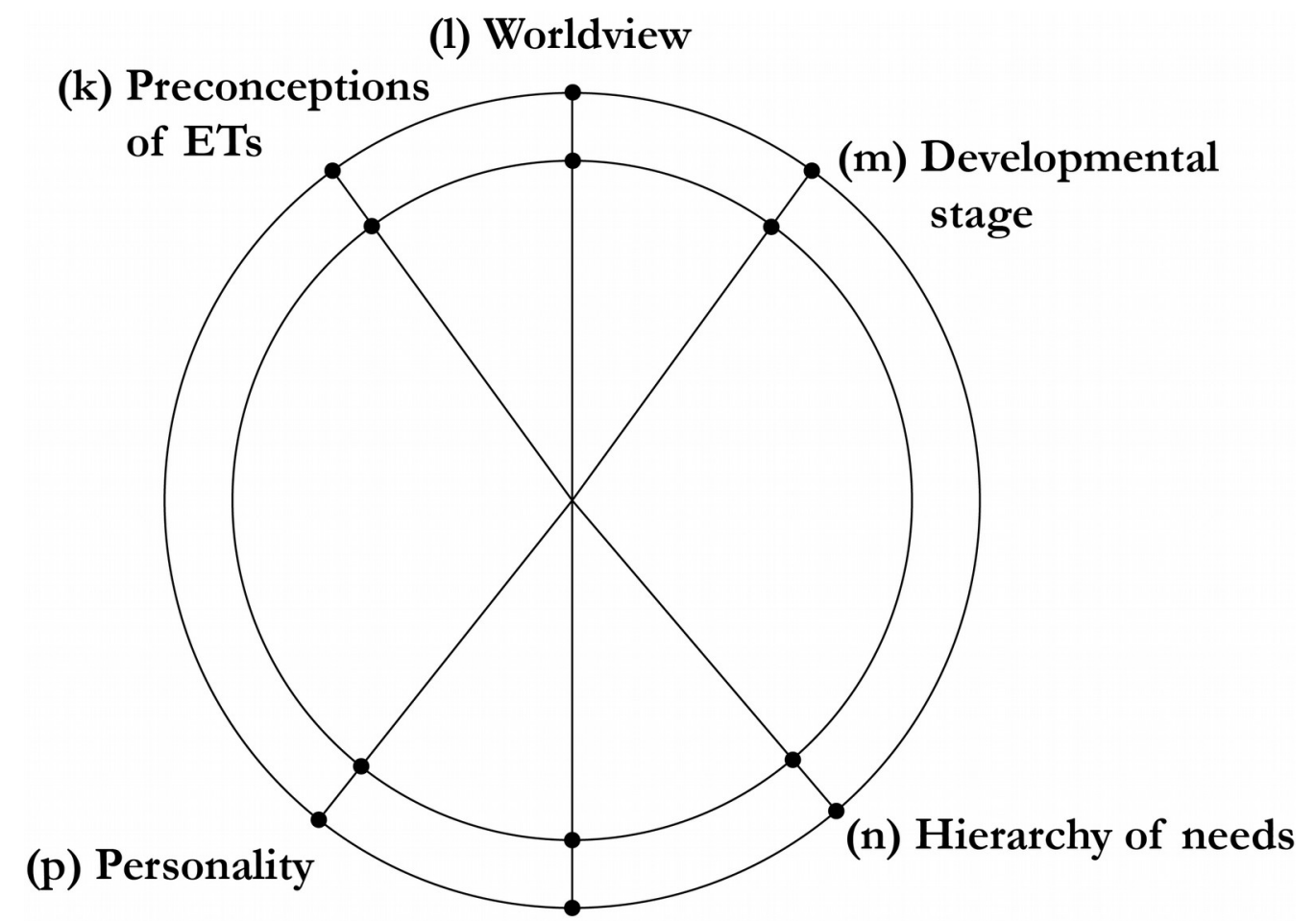

(o) Age

Figure 4 - Six dimensions of impact regarding the subjective aspects of a discovery of extraterrestrials. The options are listed in Table 1. 


\begin{tabular}{|c|c|c|}
\hline Group & Dimension & Options \\
\hline \multirow[t]{10}{*}{ Extraterrestrials } & (1) Distance & $\begin{array}{l}\text { Unknown } \\
\text { Near } \\
\text { Far }\end{array}$ \\
\hline & (2) Complexity & $\begin{array}{l}\text { Unknown } \\
\text { Simple life } \\
\text { Complex life }\end{array}$ \\
\hline & (3) Intent & $\begin{array}{l}\text { Unknown } \\
\text { Neutral } \\
\text { Benevolent } \\
\text { Malevolent } \\
\text { Benevolent \& Malevolent }\end{array}$ \\
\hline & (4) Size & $\begin{array}{l}\text { Unknown } \\
\text { Away from our scale } \\
\text { Near our scale }\end{array}$ \\
\hline & (5) Number & $\begin{array}{l}\text { One } \\
\text { Many }\end{array}$ \\
\hline & (6) Living state & $\begin{array}{l}\text { Unknown } \\
\text { Fossil } \\
\text { Dormant } \\
\text { Living }\end{array}$ \\
\hline & (7) Clarity & $\begin{array}{l}\text { Clear } \\
\text { Ambiguous }\end{array}$ \\
\hline & (8) Communicative intent & $\begin{array}{l}\text { Unknown } \\
\text { Null } \\
\text { Null, but we snoop a message } \\
\text { Limited } \\
\text { Full, transparent }\end{array}$ \\
\hline & (9) Knowledge of us & $\begin{array}{l}\text { Unknown } \\
\text { Nothing } \\
\text { Some } \\
\text { Everything }\end{array}$ \\
\hline & (10) Influence on us & $\begin{array}{l}\text { Unknown } \\
\text { None } \\
\text { Low } \\
\text { High }\end{array}$ \\
\hline \multirow[t]{5}{*}{ Humans - Objective } & (a) Universalization of knowledge & $\begin{array}{l}\text { Minimal } \\
\text { Maximal }\end{array}$ \\
\hline & (b) Speed of discovery & $\begin{array}{l}\text { Slow } \\
\text { Fast }\end{array}$ \\
\hline & (c) Data quality & $\begin{array}{l}\text { Low } \\
\text { High }\end{array}$ \\
\hline & (d) Source of discovery & $\begin{array}{l}\text { Astrobiology } \\
\text { Astroengineering / SETA } \\
\text { SETI } \\
\text { Reinterpretation of existing } \\
\text { phenomena } \\
\text { Response to active SETI }\end{array}$ \\
\hline & (e) Our knowledge of them & $\begin{array}{l}\text { Nothing except existence } \\
\text { Some } \\
\text { Everything }\end{array}$ \\
\hline
\end{tabular}




\begin{tabular}{|l|l|l|}
\hline \multicolumn{1}{|c|}{ Group } & \multicolumn{1}{|c|}{ Dimension } & \multicolumn{1}{c|}{ Options } \\
\hline Humans - Intersubjective & (f) First impression & $\begin{array}{l}\text { Positive } \\
\text { Negative }\end{array}$ \\
\hline & (g) Socio-political context & $\begin{array}{l}\text { Stable } \\
\text { Unstable }\end{array}$ \\
\hline & (h) Dissemination of the news & $\begin{array}{l}\text { Trustworthy } \\
\text { Unreliable }\end{array}$ \\
\hline & (i) World philosophy & $\begin{array}{l}\text { Western } \\
\text { Indian } \\
\text { Chinese }\end{array}$ \\
\hline Humans - Subjective & (j) Religion (k) Preconception of ETs & $\begin{array}{l}\text { Anthropocentric } \\
\text { Non-anthropocentric }\end{array}$ \\
\hline & (l) Worldview & $\begin{array}{l}\text { Near from the truth } \\
\text { Far from the truth }\end{array}$ \\
\hline & (m) Developmental stage & $\begin{array}{l}\text { No change } \\
\text { Major changes }\end{array}$ \\
\hline & (n) Hierarchy of needs & $\begin{array}{l}\text { Low } \\
\text { High }\end{array}$ \\
\hline & (o) Age & $\begin{array}{l}\text { Basic needs } \\
\text { Higher needs } \\
\text { Oounger }\end{array}$ \\
\hline & (p) Personality & $\begin{array}{l}\text { Sensitive to the discovery } \\
\text { Insensitive to the discovery }\end{array}$ \\
\hline
\end{tabular}

Table 1 - Grouped dimensions and options in preparing the impact of discovering extraterrestrials.

Although a much more comprehensive treatment would be needed, let us say a few words about these dimensions and options. Importantly, regarding ETs, I systematically include the unknown option in Table 1, because in a slow discovery, we are unlikely to know all the aspects of ETs at once.

\section{3 - Envisioning extraterrestrials}

The distance can be near or far, the nearest being in our body, e.g. if you consider genomic SETI (e.g. Davies 2010; shCherbak and Makukov 2013), or if you speculate that (directed) panspermia brought bacteria or viruses from space (e.g. Hoyle and Wickramasinghe 1990). Other near options are in our solar system, or at a 50 light years radius from Earth, which would allow a back-and-forth travel or messaging within a human lifetime. The far option includes everything beyond 50 light years, from galactic to extragalactic sources. The furthest option is if the fine-tuning of the universe itself would be a signature from an advanced civilization. This could result from a successful "search for extrauniversal intelligence" (see e.g. Pagels 1989; Gardner 2004; Steven J. Dick 2008; Vidal 2014a).

The nature of ETs can be simple or complex. Generally, the more complex, the higher the impact. The intent can be neutral (e.g. if we discover microbes), benevolent (like the E.T. in Spielberg's movie), malevolent (like almost all other movies). The intent can also be benevolent and malevolent, depending on specific contexts and situations. There is no reason that it should be Manichaean and uniform. If ten million humans were to colonize a new 
planet, they would certainly not all be benevolent with its inhabitants. The more malevolent, the higher the impact. Of course, the intent is largely a subjective dimension. Even the most malevolent ETs killing humans randomly could be interpreted by some to be a good thing that they annihilate the human race. Also, a microbe killing the Earth's biosphere might seem malevolent from our point of view, but it is neutral from its point of view.

The size of ETs can be away from our scale or near our scale. In the former case, the impact is high, because it is disconcerting if we cannot interact directly with an amazingly large galactic Type III civilization on Kardashev's (1964) scale, or with atto-sized ETs living at a very small scale (Type VI on Barrow's 1998, 133 scale; see also Vidal 2014a, chap. 9.2). Both would be equally hard to apprehend. Note that in popular culture and imagination, ETs are almost always at our scale or not far from it, but this is just a prejudice.

The number of ETs can be one or many. Finding thousands or millions of ETs will be much more distressing or exhilarating than one or two samples.

The living state of ETs could be fossil, dormant or living. The closer to the living, the higher the impact would be. Although a dormant advanced ET could also be quite intriguing or exciting, if not scary.

The dimensions of clarity, communicative intent, knowledge of us and influence on us presuppose ETI, possibly more advanced than us. If ETI communicates, the message can be clear or ambiguous. There could be many reasons for a signal or observation to be ambiguous, due to us as observers or them as transmitters, e.g. if ETs broadcast several conflicting messages (see also Harrison 1997, 202).

The communicative intent dimension is similar to the "class of phenomenon" in the Rio scale. It can be null (as I argued), or it could be null, but we snoop a message. It could also be limited in several ways. First, limited for our own good (benevolent), such as the Prime Directive; second, it could also be limited to do us harm (malevolent), like in Fred Hoyle's novel $A$ for Andromeda. It could also be limited for specific purposes such as beacon, navigation, which would be a more neutral message, omnidirectional, not Earth-specific. We could also imagine a full, transparent communicative intent, even if this does not guarantee full mutual comprehension. For more on the diversity of signal detection scenarios, see e.g. (Tarter 1992).

Their knowledge of us could amount to nothing, but they could know some things or even nearly everything about us, if they had been observing the Earth for billions of years. To the limit, their knowledge of us could even have turned into an influence upon us. Although it sounds like material for science fiction or ufology, it remains a possibility that ETs have or had a low or high influence on us (consider e.g. panspermia or directed panspermia).

\section{4 - Envisioning humanity's reaction: objective dimensions}

Let us now turn to the 16 dimensions that are key to understand the impact on humanity (Fig. 3-4). They are classified in three categories: objective, subjective and intersubjective categories which have proven their worth in the history of ideas (Vidal 2012).

The main 'objective' or scientific impact of finding ETs will be to literally universalize our knowledge. For now, only physics and some chemistry have been proven to hold beyond the confine of our atmosphere. As Table 2 illustrates, all other domains of knowledge remain to be universalized. However, thinking in an astrobiological or cosmological context, we have 
already started to universalize many domains of knowledge. If we find ETs, the more domains of knowledge become universalized, the greater the impact. The minimal impact here is that biology will be universalized once we find life beyond Earth. It would already be an immense breakthrough, but at the limit, the universalization of knowledge could be maximal, as some astrobiologists have explored (see references in Table 2).

\begin{tabular}{|l|l|l|}
\hline \multicolumn{1}{|c|}{ Universal } & \multicolumn{1}{|c|}{ Comments } & \multicolumn{1}{c|}{ References (e.g.) } \\
\hline Physics & We have it! & $\begin{array}{l}\text { Physics and astrophysics } \\
\text { textbooks }\end{array}$ \\
\hline Biology & $\begin{array}{l}\text { Living system theory, } \\
\text { universal biology }\end{array}$ & $\begin{array}{l}\text { (Miller 1978; Freitas Jr 1981; } \\
\text { Schulze-Makuch and Irwin } \\
\text { 2008) }\end{array}$ \\
\hline Language & Anticryptography & (Freudenthal 1960) \\
\hline Sociology & Astrosociology & (Freitas Jr 1979) \\
\hline Economy & Thermoeconomics & (Corning 2005) \\
\hline Ethics and laws & $\begin{array}{l}\text { Thermoethics } \\
\text { Thermo-evo-devo ethics } \\
\text { Celegistics }\end{array}$ & $\begin{array}{l}\text { (Freitas Jr 1979) } \\
\text { (Vidal 2014a, chap. 10) } \\
\text { (Vakoch 2014) }\end{array}$ \\
\hline Aesthetics & What is beautiful for an ET? & (Freitas Jr 1979, sec. 22.5) \\
\hline Theology & Cosmotheology & $\begin{array}{l}\text { (Steven J. Dick 2000; Peters } \\
\text { 2014) }\end{array}$ \\
\hline Culture & Cosmos and culture & $\begin{array}{l}\text { (Steven J. Dick and Lupisella } \\
\text { 2009) }\end{array}$ \\
\hline Eschatology & Facing cosmic doom & (Vidal 2014a; Vidal 2014b) \\
\hline
\end{tabular}

Table 2 - Examples of domains of knowledge which remain to be universalized. Reflecting in in an astrobiological or cosmological context, some authors have already started to think in truly universal terms.

I argued that the speed of discovery will be slow, but I may turn out to be wrong. Compare for example intercepting a message in plain English versus a cryptic message that takes us 20 years to decode. The most likely scenario is that opinions will oscillate with strong arguments sometimes supporting, sometimes refuting the claim of discovery. The faster the scientific community converges, the higher the impact will be. This dimension is similar to the credibility factor in the Rio scale.

The data quality we gather could be low or high depending on whether it is steady or transient, complete or incomplete, clear or ambiguous. The clearer, more complete and steadier, the higher the quality it will be and the more seriously the discovery will be taken.

The source of discovery could come from mainstream astrobiology, searching for life in our solar system, or hunting Earth-like planets. It could also come from astroengineering, or search for extraterrestrial artefacts (SETA), or SETI as practiced by the SETI institute. But it could also be a reinterpretation of existing phenomena. For example, if some UFO reports would end up convincing the scientific community. Importantly, authors have argued that ETs could already be in our astrophysical data (Shvartsman, cited in Heidmann and Klein 1991, 390:393; Davies 2010, 124; Vidal 2014a, 205). Should the scientific community be ashamed if ETs would turn out to already have been in our data? I don't think so, and previous scientific revolutions support this view. Newton's or Darwin's contributions were not in the 
discovery of new gravitational bodies or new living species. Their contribution was to propose new theories to explain and interpret a variety of already known gravitational phenomena and already known living species. Finally, if we would have a reply to one of the few messages we sent to space, i.e. a response to active SETI, the impact would certainly be extremely exciting.

Our knowledge of them could be minimal, for example if we discover a cryptic message, we would know nothing except their existence. We could know some more, or even nearly everything about them. Note that this dimension needs to be compared with their knowledge of us (9): the more asymmetry in our disfavor, the more distressing it would be for us.

\section{5 - Envisioning humanity's reaction: intersubjective dimensions}

Turning to the intersubjective or social group of dimensions, the first impression is crucial, because it could lead to butterfly effects (Harrison 1997, 199; Tarter 1992). Indeed, cycles of interactions, depending on whether they start on good or bad terms, could bifurcate either towards friendly relationships or to fearful ones. Overall, the first impression can thus be positive or negative.

Whether the socio-political context is stable or unstable will also play an important role, especially in forming the first impressions and in shaping the first reactions (Harrison 1997). An initial and promising discovery may also lead to a symbolic cold war to secure the discovery, like the race between the soviets and the USA to fly to the Moon.

The dissemination of the news can be transparent, trustworthy, following existing recommendations and protocols. But it could as well become unreliable, triggering rumors, disbeliefs and confusion (Harrison 1997, 206-213).

Academia is strongly influenced by a Western world philosophy, but as comparative philosophers have observed (e.g. Bahm 1995; Smith 1957), there are obviously other major world philosophies to take into account, such as the Indian and the Chinese ones. This is especially important to take into account if we want to study the global impact, considering the large world population which holds a non-western philosophy.

How will various religious groups react? As previous studies have shown (e.g. Vakoch and Lee 2000), the religious affiliation matters less than a belief in anthropocentrism o r non-anthropocentrism. Still, given how important the influence of religions is to billions of people, we must study how religious belonging would affect the impact. Specific cases may be important to monitor, such as the reactions of UFO religions.

\section{6 - Envisioning humanity's reaction: subjective dimensions}

In the subjective group (Fig. 4), the single most important dimension is our preconception of ETs (Connors 1976). Depending on whether we were near from the truth or far from the truth, reactions will vary immensely. This dimension can thus be assessed differentially, by drawing two versions of the spider diagram in Fig. 2 (the dimensions 1-10). One version is how we imagine ETs to be, and the second version is how ETs really are, to be drawn at the time of discovery. If the two spider diagrams overlap perfectly, it means that we were spot on, and the discovery will not surprise us. But if we turn out to 
be wrong in all options of the 10 dimensions, it will be a big surprise if not a psychological shock.

The dimension of worldview assesses if the discovery leads to no change in worldview, or to major changes. Two worldview models can be used for this purpose. First, using the collated model of Koltko-Rivera (2004), which is composed of 42 dimensions collated in groups such as human nature, will, cognition, behavior or truth. This model proposes a total of 119 options, to be chosen in the various dimensions. Again, the impact on worldviews can be assessed differentially, before and after the discovery of ETs. The worldview impact is the difference between your current worldview and the new worldview that finding ETs implies. If your worldview wouldn't change for any ET detection scenario (dimensions 1-10), it means you're truly ready. But double check that you are not in denial, rationalizing and protecting your old worldview. A direct (non-differential) assessment would require confronting the whole landscape of worldviews with the landscape of possible detections of ETs. The other worldview model is the one of Leo Apostel (Aerts et al. 1994; Vidal 2008). Here, a worldview is defined as answers to big questions such as: Where does it all come from? Where are we going? What is good and what is evil? What is true and what is false? This model allows to ask: Does the discovery of ETs change your views on the origin of the universe? On the future? On morality? On the nature of knowledge? Here we can contrast the London scale with the present approach, taking the example of panspermia. On the London scale, the scenario scores relatively low (1.4 to 2.8 out of 10) (Almár and Race 2011, 691). However, if proven true, panspermia would require to re-write our worldview answer to "where does it all come from?", regarding the origin of life on Earth. For many people, such a discovery would come as a shock, because it would refute the story of cosmic evolution that most hold today. Note that a disconfirmation of a worldview can be both transformative or catastrophic.

Psychologists have shown that as humans grow, their motor skills, cognitive abilities, moral reasoning, emotional coping mechanisms, and their self-concept develop in stages (e.g. Piaget 1954; Kohlberg 1984; Laske 2008). Different people at different stages of development will certainly react differently to the discovery of ETs. This approach is especially useful to study, because developmental theories aim to relate many psychological dimensions, from feelings and motivation, to ethics and learning strategy (e.g. Graves 1974). People in lower stages will react differently than people in higher stages. Note that one effect of the discovery could also trigger changes in developmental stage. Generally, a person grows up in stages, simply because higher stages are more adaptive, allowing to successfully deal with more or more complex situations. Even if the worldview change is catastrophic at first, hopefully the discovery of ETs could later become transformative and trigger profound psychological changes to higher stages.

A robust psychological theory is Maslow's (1954) hierarchy of needs. Which level or levels of Maslow's hierarchy would the discovery affect? It could affect basic needs, or higher needs. For example, an ET microbe which would make all our food poisonous would be a disaster for everyone, affecting physiological and safety needs of the whole planet. By contrast, the discovery of a lost civilization, with no impact on daily behavior and functioning would only impact people who wonder about the position of mankind in the universe. Almost all scenarios would not impact basic human needs. In this pragmatic sense, we can argue that most potential discoveries (especially those far from 
Earth) will have a weak impact on a vast majority of people, because they won't need to change their behavior.

People of younger o r older age will react differently. Younger generations are generally more progressive, more open to new experiences and discoveries. They are likely to be more open and more affected by the discovery, while older generations might either deny the discovery or try to make it fit with their older worldview, instead of changing it radically. Note that the "age" dimension overlaps with the "developmental stage" dimension.

Different personality types will be more or less sensitive or insensitive to the discovery. Although personality and worldview overlap, little research has been done on this topic (Koltko-Rivera 2004, 44), so it make sense to consider personality as a dimension on its own. Harrison (1997, 245-248) discussed various personality traits which would affect human reactions. For example, the emotional stability (how well a person copes with stress), the rigidity or dogmatism of his worldview (how hard it is to change it), the default affectivity stance (positive and cheerful, versus negativistic and anxious), as well as the self-esteem (low or high). Obviously, someone emotionally stable, flexible in his worldview, positively minded, and with a high self-esteem will react much better than someone with the opposite attributes.

\section{7 - Discussion}

Let us discuss our dimensions and options. Regarding ETs, dimensions 1-3 are the most important parameters. As Harrison $(1997,237)$ put it: "If we believe that their technology is not much better than ours, that they are safely sequestered in some remote corner of the universe, and that they are basically "good guys", we will feel less threatened than if they have an overwhelming technical superiority, are already in our neighborhood, and seem unfavorably disposed toward humans".

Regarding the human side, I selected dimensions (a-p) relevant for the question of impact. Assessing the impact on us presupposes that we have a model of humanity. This is of course extremely hard to do, and would require nothing less than a synthesis of social sciences. No modeling is neutral and purely objective. Modeling always has a goal (Sterman 2000). What is the goal that we pursue when we try to model impacts of discovering ETs? Different goals will require different models, focusing on different dimensions. My own goal and hope is that these two sets of dimensions will prove useful as a conceptual framework and repository for researchers and policy makers to explore the landscape of impacts related to the discovery of ETs. One could choose to focus on studying the impact on a common profile of most people, or some specific categories of people (e.g. decision makers, leaders), or on different human activities (academia, religions, politics, public health, economics, etc.).

Focusing on the impact on humanity may also be too restrictive for a whole class of scenarios where ETL affects not only the human species, but also the whole ecosystem. An example would be a bacterial colony coming from a meteorite and contaminating and disturbing the whole ecosystem.

Mark Neal (2014) recently reviewed the question of ETs in the context of risk management. There are of course many risks we can imagine, but what if the discovery is also a great opportunity to grow and learn? Is there a field of research such as "opportunity management"? If one wants to stick with risk management, then we need to consider the risk of not making the most of the 
opportunities. For now, we have the opportunity to prepare for discovery, which means to explore an immense landscape of possibilities. In the end, we may learn more now that we are free to explore the landscape of possibilities, rather than after the actual discovery, which would put constraints on the landscape. The astrobiological journey may be more important than the destination.

Does the model have too many dimensions? Multiplying the options, we see that there are 172800 possibilities (i.e. spider diagrams) for ETs, and 442368 on the side of humanity. Putting the two together, this totals to more than 76 billion scenarios to study! With Koltko-Rivera (2004, 28), I'd argue that it's a better approach to start with too many dimensions rather than too few:

Ultimately, multivariate empirical research will reveal which dimensions are actually distinct from one another. Put crudely: "Factor analyze them all-let eigenvalues sort them out." If one starts out with a detailed set of dimensions, research may reveal a simpler structure. Starting out with too small a set of dimensions would make it difficult or impossible to determine whether a more complex approach is needed. Whether tailoring clothing or theory, it is wise to start with more material than the finished product will require.

Further work needs to be done to tailor the model I proposed. As we mentioned, developmental psychology theorists may see patterns at different stages of cognitive, emotional and moral development, and thus could reduce the space of human reactions. An other approach would be to reduce the state space thanks to decision trees (see Billingham 2002). For example, if bacteria are found on Mars, we don't need to consider the parameters of communicative intent.

Preparing for this major discovery, educating people to cosmic evolution and a wide array of possible detections and reactions is the most useful thing we can do now. If people entertain a cosmic culture, they will be much more ready to absorb the news that we are not alone in the universe.

\section{5 - Conclusion}

I first argued that humans are unlikely to meet peers, and more likely to discover either microbial life or Kardashev Type II stellar civilization. Those two encounters will be non-communicative, as such extraterrestrials would be either immensely inferior or immensely superior to us.

I then argued that the discovery will be slow, as arguments and counterarguments will likely be difficult to resolve conclusively, leading to waning public and media interest, and possibly an anti-climatic announcement when a discovery is actually confirmed.

Finally, getting ready for impact means that a smooth absorption should be our goal. This can be achieved by remaining aware of the wide array of possible extraterrestrials, and human reactions. To this end, I introduced a new multidimensional impact model covering both what extraterrestrials would be like and the possible socio-psychological reactions of humans.

Anticipating and preparing for every single scenario out of the 76 billion entailed by the model is clearly preposterous (see also Harrison 2007, ch. 9). But it would certainly be valuable to explore this space of possibilities in more depth and identify the most hazardous ones for human psyche and society. 
Taking even wider perspectives, we should also care beyond humanity, to also preserve the Earth ecosystem. Looking at the other side of the impact, we should also study our impact on possible ecosystems at the scales of the solar system, the galaxy or the universe.

I hope this model will provide a useful framework for researchers and policy makers to explore and appreciate the diversity and complexity of possible impacts.

\section{6 - Acknowledgments}

I thank Mark Lupisella, John Trapaghan, Steven J. Dick, Cadell Last, and Albert A. Harrison for helpful corrections and discussions.

\section{7 - References}

Aerts, D., L. Apostel, Bart De Moor, Staf Hellemans, Edel Maex, Hubert Van Belle, and Jan Van der Veken. 1994. World Views. From Fragmentation to Integration. VUB Press. http://www.vub.ac.be/CLEA/pub/books/worldviews.pdf.

Almár, Iván. 2001. "How the Rio Scale Should Be Improved." In IAA-01-IAA.9.2.03. International Academy of Astronautics. http://avsport.org/IAA/abst2001/rio2001.pdf.

2011. "SETI and Astrobiology: The Rio Scale and the London Scale." Acta Astronautica 69 (9-10): 899-904. doi:10.1016/j.actaastro.2011.05.036.

Almár, Iván, and Margaret S. Race. 2011. "Discovery of Extra-Terrestrial Life: Assessment by Scales of Its Importance and Associated Risks." Philosophical Transactions of the Royal Society A: Mathematical, Physical and Engineering Sciences 369 (1936): 67992. doi:10.1098/rsta.2010.0227.

Bahm, Archie J. 1995. Comparative Philosophy: Western, Indian, \& Chinese Philosophies Compared. Revised edition. World Book.

Barrow, J. D. 1998. Impossibility: The Limits of Science and the Science of Limits. Oxford University Press, USA.

Beech, Martin. 2008. Rejuvenating the Sun and Avoiding Other Global Catastrophes. Springer.

Billingham, John. 2002. "Pešek Lecture: SETI and Society-decision Trees." Acta Astronautica 51 (10): 667-72. doi:10.1016/S0094-5765(02)00023-1.

Bradbury, Robert J., Milan M Ćirković, and George Dvorsky. 2011. "Dysonian Approach to SETI: A Fruitful Middle Ground?" Journal of the British Interplanetary Society 64: 156-65.

Connors, Mary M. 1976. The Role of the Social Scientist in the Search for Extraterrestrial Intelligence. NASA Ames Research Center.

Corning, P. A. 2005. Holistic Darwinism: Synergy, Cybernetics, and the Bioeconomics of Evolution. University of Chicago Press.

Davies, P. C. W. 2010. The Eerie Silence: Are We Alone in the Universe?. London: Penguin Books.

Dick, Steven J. 1996. The Biological Universe: The Twentieth Century Extraterrestrial Life Debate and the Limits of Science. Cambridge University Press. 2000. "Cosmotheology: Theological Implications of the New Universe." In Many Worlds. The New Universe, Extraterrestrial Life and the Theological Implications, edited by S. J. Dick, 191-210. Philadelphia and London: Templeton Foundation Press.

2008. "Cosmology and Biology." In Proceedings of the 2008 Conference on the Society of Amateur Radio Astronomers. June 29 - July 2, 1-16. National Radio Astronomy Observatory, Green Bank, West Virginia: Amateur Radio Relay League. http://evodevouniverse.com/uploads/f/f3/Dick 2008 - Cosmology and Biology.pdf. 2013. Discovery and Classification in Astronomy: Controversy and Consensus. New york, NY, USA: Cambridge University Press.

Dick, Steven J., and Mark L. Lupisella, eds. 2009. Cosmos and Culture: Cultural Evolution in a Cosmic Context. Washington D.C.: Government Printing Office, NASA SP-20094802. http://history.nasa.gov/SP-4802.pdf. 
Dyson, F. J. 1960. "Search for Artificial Stellar Sources of Infrared Radiation." Science 131 (3414): 1667-68. doi:10.1126/science.131.3414.1667.

1966. "The Search for Extraterrestrial Technology." In Perspectives in Modern Physics, edited by R.E. Marshak, 641-55. New York: John Wiley \& Sons.

Freitas Jr, R. A. 1979. Xenology: An Introduction to the Scientific Study of Extraterrestrial Life, Intelligence, and Civilization. Xenology Research Institute (1979-2010). http://www.xenology.info/Xeno.htm.

__- 1981. "Xenobiology." Analog Science Fiction/Science Fact 101: 30-41. http://www.xenology.info/Papers/Xenobiology.htm.

Freitas Jr, R. A., and Francisco Valdes. 1985. "The Search for Extraterrestrial Artifacts (SETA)." Acta Astronautica 12 (12): 1027-34. doi:10.1016/0094-5765(85)90031-1.

Freudenthal, H. 1960. Lincos: Design of a Language for Cosmic Intercourse. North-Holland.

Gardner, James N. 2004. "The Physical Constants as Biosignature: An Anthropic Retrodiction of the Selfish Biocosm Hypothesis." International Journal of Astrobiology 3 (03): 229-36. doi:10.1017/S1473550404002162.

Graves, C. W. 1974. "Human Nature Prepares for a Momentous Leap.” The Futurist 8 (2): 72 85.

Harrison, Albert A. 1997. After Contact: The Human Response To Extraterrestrial Life. New York: Plenum Trade. 2007. Starstruck: Cosmic Visions in Science, Religion and Folklore. Berghahn Books.

Heidmann, Jean, and Michael J. Klein, eds. 1991. Bioastronomy The Search for Extraterrestial Life - The Exploration Broadens. Vol. 390. Berlin, Heidelberg: Springer Berlin Heidelberg.

Hoyle, Fred, and N. C. Wickramasinghe. 1990. Cosmic Life-Force. 1st American ed. New York: Paragon House.

Kardashev, N. S. 1964. "Transmission of Information by Extraterrestrial Civilizations." Soviet Astronomy 8 (2): 217-20. http://adsabs.harvard.edu/abs/1964SvA.....8..217K.

Kohlberg, Lawrence. 1984. The Psychology of Moral Development: The Nature and Validity of Moral Stages. 1st ed. Harpercollins College Div.

Koltko-Rivera, Mark E. 2004. "The Psychology of Worldviews." Review of General Psychology 8 (1): 3-58. doi:10.1037/1089-2680.8.1.3.

Kuhn, T. S. 1957. The Copernican Revolution; Planetary Astronomy in the Development of Western Thought. Cambridge: Harvard University Press.

1970. The Structure of Scientific Revolutions. University of Chicago Press Chicago.

Laske, Otto E. 2008. Measuring Hidden Dimensions of Human Systems: Foundations of Requisite Organization. 1st ed. Medford, MA: IDM Press SAN 257-9618.

Maslow, Abraham H. 1954. Motivation and Personality. 1st ed. Harper's Psychological Series. New York: Harper.

McKay, David S., Everett K. Gibson, Kathie L. Thomas-Keprta, Hojatollah Vali, Christopher S. Romanek, Simon J. Clemett, Xavier D. F. Chillier, Claude R. Maechling, and Richard N. Zare. 1996. "Search for Past Life on Mars: Possible Relic Biogenic Activity in Martian Meteorite ALH84001." Science 273 (5277): 924-30. doi:10.1126/science.273.5277.924.

Miller, J. G. 1978. Living Systems. McGraw-Hill New York.

Neal, Mark. 2014. "Preparing for Extraterrestrial Contact." Risk Management 16 (2): 63-87. doi:10.1057/rm.2014.4.

Pagels, Heinz R. 1989. The Dreams of Reason. New York: Bantam.

Peters, Ted. 2014. "Astrotheology: A Constructive Proposal." Zygon ${ }^{\circledR} 49$ (2): 443-57. doi:10.1111/zygo.12094.

Piaget, Jean. 1954. The Construction of Reality in the Child. New York: Basic Books.

Schulze-Makuch, Dirk, and Louis N. Irwin. 2008. Life in the Universe: Expectations and Constraints. 2nd ed. Advances in Astrobiology and Biogeophysics. Berlin ; London: Springer.

shCherbak, Vladimir I., and Maxim A. Makukov. 2013. "The 'Wow! Signal' of the Terrestrial Genetic Code.” Icarus 224 (1): 228-42. doi:10.1016/j.icarus.2013.02.017.

Smith, Huston. 1957. "Accents of the World's Philosophies." Philosophy East and West 7 (1/2): 7-19.

Sterman, J.D. 2000. Business Dynamics: Systems Thinking and Modeling for a Complex World. Irwin McGraw-Hill.

Tarter, Donald E. 1992. "Interpreting and Reporting on a SETI Discovery: We Should Be Prepared." Space Policy 8 (2): 137-48. doi:10.1016/0265-9646(92)90037-V. 
Vakoch, Douglas A., ed. 2014. Extraterrestrial Altruism: Evolution and Ethics in the Cosmos. The Frontiers Collection. Springer Berlin Heidelberg.

Vakoch, Douglas A., and Y. -S Lee. 2000. "Reactions to Receipt of a Message from Extraterrestrial Intelligence: A Cross-Cultural Empirical Study.” Acta Astronautica 46 (10-12): 737-44. doi:10.1016/S0094-5765(00)00041-2.

Vidal, C. 2008. "What Is a Worldview? Published in Dutch as: 'Wat Is Een Wereldbeeld?." In Nieuwheid Denken. De Wetenschappen En Het Creatieve Aspect Van De Werkelijkheid, edited by Hubert Van Belle and Jan Van der Veken, 71-85. Leuven: Acco. http://cogprints.org/6094/.

2012. "Metaphilosophical Criteria for Worldview Comparison." Metaphilosophy 43 (3): 306-47. doi:10.1111/j.1467-9973.2012.01749.x. http://homepages.vub.ac.be/ clvidal/writings/Vidal-Metaphilosophical-Criteria.pdf 2014a. The Beginning and the End - The Meaning of Life in a Cosmological Perspective. The Frontiers Collection. Springer. http://arxiv.org/abs/1301.1648.

2014b. "Cosmological Immortality: How to Eliminate Aging on a Universal Scale." Current Aging Science. doi:10.2174/1874609807666140521111107. http://homepages.vub.ac.be/ clvidal/writings/Vidal-Cosmological-Immortality.pdf

Vilenkin, Alexander. 2011. "The Principle of Mediocrity." Astronomy \& Geophysics 52 (5): 5.33-35.36. doi:10.1111/j.1468-4004.2011.52533.x.

Wainwright, Milton. 2014. "A Presumptive Fossilized Bacterial Biofilm Occurring in a Commercially Sourced Mars Meteorite." Journal of Astrobiology \& Outreach 02 (02). doi:10.4172/2332-2519.1000114.

Wright, J. T., R. L. Griffith, S. Sigurdsson, M. S. Povich, and B. Mullan. 2014. "The G Infrared Search for Extraterrestrial Civilizations with Large Energy Supplies. II. Framework, Strategy, and First Result." The Astrophysical Journal 792 (1): 27. doi:10.1088/0004-637X/792/1/27. 